\title{
Exploring finite-size effects in strongly correlated systems
}

\author{
M. Buraczynski ${ }^{1}$, W. Dawkins ${ }^{1}$, N. Ismail ${ }^{1}$, and A. Gezerlis ${ }^{1}$ \\ ${ }^{1}$ Department of Physics, University of Guelph, Guelph, On N1G 2W1, Canada
}

\begin{abstract}
Complexities greatly limit any study of strongly correlated systems to a small number of particles. Thus, any attempt at understanding infinite systems such as those arising from neutron matter (NM) must consider finite-size (FS) effects at play when below the thermodynamic limit (TL). In these conference proceedings we provide some examples of FS effects at work and discuss our prescription for extrapolating the physics of extended systems. We present our methodology and calculations performed for an assortment of strongly correlated (SC) systems. Ab initio, non-perturbative Quantum Monte Carlo (QMC) methods can be employed to accurately compute ground-state energies and finite-temperature properties. We apply these to periodically modulated NM and use our results to constrain phenomenological theories of nuclei and study the static response of NM.
\end{abstract}

\section{Introduction}

Strongly interacting systems in quantum mechanics are those in which the interaction energy among constituents is significant, i.e. comparable to the kinetic energy. These are much harder to understand than systems with weak interactions. The latter can be well approximated by free particles in an external potential and thoroughly analyzed using well-established methods such as perturbation theory. Unfortunately, correlations in strongly interacting systems are complicated and cannot be tackled using traditional techniques that work for weakly interacting systems. Investigations are generally limited to few particles because of complexities introduced by the interactions. Thus it is impossible to directly compute macroscopic properties of such systems using modern computational technology. Nevertheless, understanding the relationship between finite and thermodynamic limit (TL) physics may allow an extrapolation to such scales. The exploration of finite-size (FS) effects in strongly correlated (SC) systems is therefore highly important and very rewarding.

There is no shortage of SC systems in low-temperature physics. They appear in nuclear, condensed matter, solid-state, and cold-atom physics [1]. Many superconductors are SC [2]. Neutron matter (NM) is a SC system as well [3,4]. The nuclear force is strong and especially complicated. There are central, spin, and tensor terms to name a few. Neutrons do not form bound systems by themselves so they are easier to study than combinations of protons and neutrons. They are also relevant to actual physics: they appear in large quantities in neutron stars and are the main constituents of neutron-rich nuclei [3]. The physics of NM is consequential to neutron-star structure. Its importance is recognized and much research continues to investigate and improve our understanding of its equation of state (EOS) [5-10]. We present our contribution here on the response of NM to an external sinusoidal potential $[11,12]$. Not only does this provide feedback on the nature of pure NM 


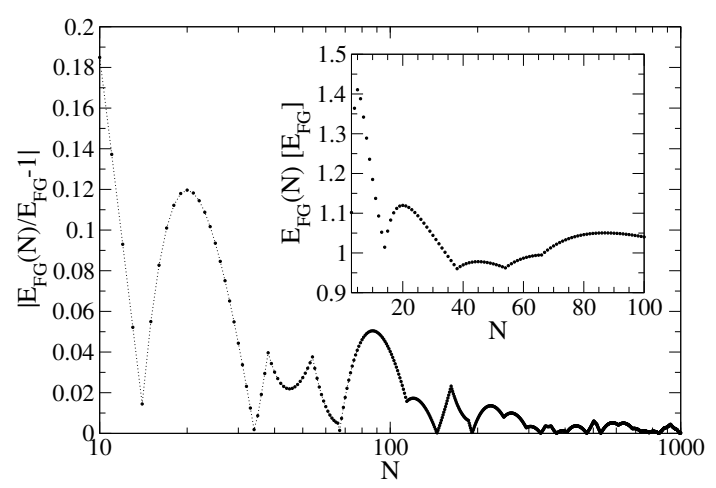

Figure 1. Convergence in energy to the TL for a non-interacting free-Fermi gas. The energy is plotted in units of $E_{F G}$ which is the energy per particle at the TL. Re-plotted and inset using a linear scale for $N$ [12].

through the static response functions, it is also a better comparison to naturally occurring physics than homogeneous NM. The density of neutrons is not constant in most neutron-rich systems. This inhomogeneity arises in neutron-star crusts because the matter contains a lattice of nuclei that perturbs the unbound neutrons. Nuclei are inhomogeneous because the density of nucleons decreases towards the edge of the nucleus. The connection to nuclei from NM is made through various energy density functionals (EDFs) that are fit to masses and radii of nuclei [13-16]. The NM EOS can be used as a constraint as well [14, 17-22]. We use our NM results as an input constraint for EDFs of the Skyrme type $[11,12]$.

Calculations for SC systems have greatly benefitted from modern advancements in computation. Today's supercomputers allow for simulations of sizable numbers of particles using methods that are derived from first principles. The non-perturbative family of Quantum Monte Carlo (QMC) methods are excellent for tackling a wide variety of complicated problems. We apply Auxiliary Field Diffusion Monte Carlo (AFDMC) to calculate ground-state energies for our neutron system. This is a projector method that extracts the ground-state from a trial wave function and is especially suited for handling complicated spin correlations arising from the nuclear interaction. Of course these simulations are largely limited by particle number. In what follows we discuss the consequences of FS on quantum systems and apply our observations to NM.

\section{Finite-size effects for non-interacting fermions}

\subsection{The free-Fermi gas}

The natural starting point for understanding FS effects is to consider non-interacting particles and explore FS in a free-gas of fermions. We confine $\mathrm{N}$ particles to a cubic box of side length $L$ and impose periodic boundary conditions on the wave function. This respects translational invariance as one can imagine identically occupied cubes filling up all of space for an extended system in the TL. The energy eigenstates of a free-Fermi gas are plane waves with wave vectors $\mathbf{k}=(2 \pi / L)\left(n_{x}, n_{y}, n_{z}\right)$, where $n_{x}, n_{y}, n_{z}$ are integers. At $T=0$ the lowest available energy levels are occupied. The energy is given by $E=\hbar^{2} \mathbf{k}^{2} / 2 m$. Given spin there is an additional degeneracy for each spin-projection state.

The macroscopic system/TL can be envisioned by iteratively adding particles at a fixed density by increasing the box length $L$ accordingly. In this limit intensive properties like energy per particle 


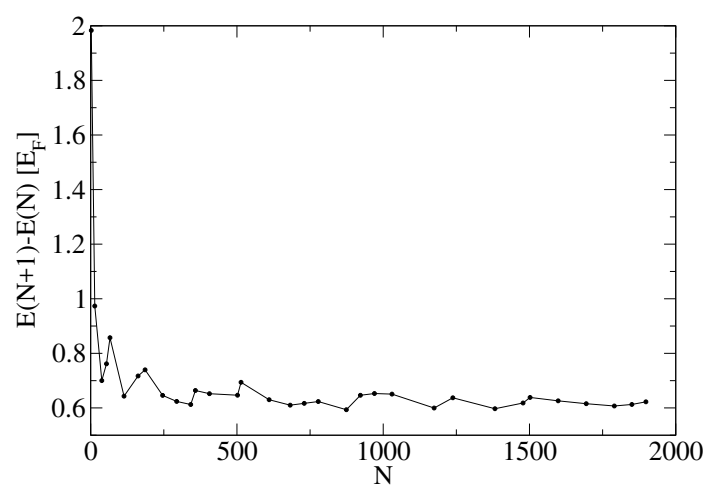

Figure 2. Energy difference from adding a single particle to $N$ particles at a fixed density of $0.05 \mathrm{fm}^{-3}$.

converge to a macroscopic value. It can be analytically shown that the energy per particle converges to $E_{F G}=(3 / 5) E_{F}$, where $E_{F}$ is the Fermi energy. This is shown in Fig. 1 where the ratio of energy per particle to $E_{F G}$ converges to 1 as $N$ goes to infinity. Any difference between a finite system and the TL is called a FS effect. The FS effects in Fig. 1 are quantified as $\left|E_{F G}(N) / E_{F G}-1\right|$ and are larger at smaller particle numbers. The cusps in the inset occur near closed shells where all occupied energy levels are filled to capacity. The wave function is ambiguous whenever an occupied energy level is not completely filled because only a subset of degenerate states are occupied and this subset is not unique. For this reason it is usually preferred to study closed shells. Closed shell configurations for the free-Fermi gas occur at $N=\{2,14,38,54,66,114 \ldots\}$. The FS effects in Fig. 1 appear small near the $N=66$ closed shell. This motivated us to choose 66 particles in our NM study.

It's also interesting to explore the energy cost associated with adding an extra particle. At large $N$ we expect $E(N+1)-E(N) \approx E_{F G}$. Fig. 2 displays this convergence at a density of $0.05 \mathrm{fm}^{-3}$. Note that increasing $N$ implies increasing the box length $L$. Wave vector magnitudes decrease with increasing $L$ because $\mathbf{k}$-space is reciprocal to physical space. This implies that the energy of $N$ particles decreases as extra particles are added at fixed density. Since the energy of an additional particle is $\approx E_{F}$ when $N$ is large, this implies that the $N$ particle energy change is $E_{F G}-E_{F}=-(2 / 5) E_{F}$.

Calculations for a non-interacting gas are trivial compared to a strongly interacting gas. Energies can be computed for thousands of particles. This allows us to determine TL properties of noninteracting particles in a one-body potential. Our prescription for extrapolating an interacting system to the TL uses the TL energy of a non-interacting gas:

$$
E_{\mathrm{I}}(\infty)=E_{I}(N)-E_{N I}(N)+E_{N I}(\infty)
$$

Table 1. SLy4 energy per particle for 66 neutrons (first column) in a box at various densities. The second column contains the extrapolated results computed using Eq. 1. The final column has known SLy4 energies in the TL [12].

\begin{tabular}{lccc}
\hline$n\left(\mathrm{fm}^{-3}\right)$ & $E_{I}(66)(\mathrm{MeV})$ & $E_{I}(\infty)(\mathrm{MeV})$ & $\mathrm{TL}(\mathrm{MeV})$ \\
\hline 0.04 & 7.15 & 7.22 & 7.23 \\
0.06 & 8.62 & 8.71 & 8.73 \\
0.08 & 9.99 & 10.10 & 10.13 \\
0.10 & 11.40 & 11.53 & 11.58 \\
\hline
\end{tabular}




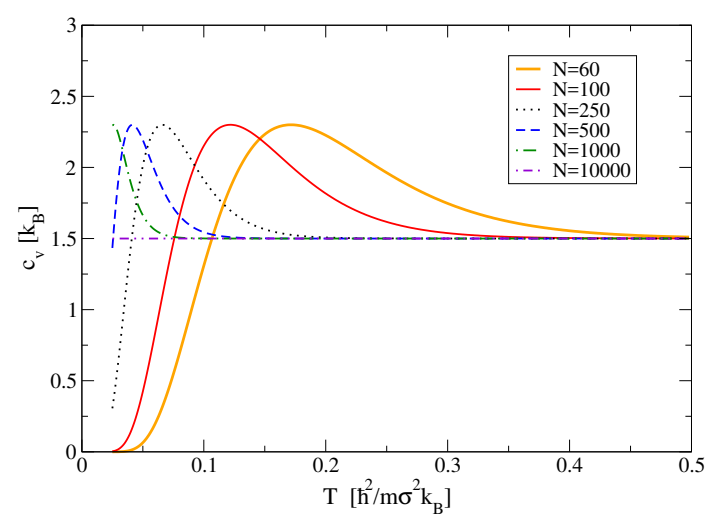

Figure 3. Specific heat of a non-interacting gas versus temperature temperature (constant density, $n \sigma^{3}=0.2063$ ). For large particle numbers $N$, the observed spike disappears [23].

where $E_{I}$ and $E_{N I}$ are energies per particle for the interacting and non-interacting systems respectively. Eq. 1 extrapolates to the TL by adding a non-interacting correction to the energy of interacting particles. We applied Eq. 1 to local density approximation (LDA) calculations of homogeneous neutron matter. These are calculations that use EDFs to compute the ground-state energy. The SLy4 parametrization of the Skyrme EDF was used. [14] The energy per particle of 66 neutrons at various densities are listed in Table 1. They agree to within $0.5 \%$ with known SLy4 energies (final column).

In related recent work, we have also extended the study of FS effects to the case of finite temperature [23]. There, assuming classical statistics and quantum mechanics, the energy is computed via the averaging of the energies over a Maxwell-Bolztmann distribution. This was carried out at a constant density of $n \sigma^{3}=0.2063$ for several particle numbers ( $\sigma$ is here just a length scale, which would correspond to the hard-sphere diameter if interactions were turned on). By taking the derivative of $E / N$ with respect to temperature, we have calculated the specific heat for this system, Fig. 3. This shows a convergence to the equipartition theorem value (i.e., 3/2) at increasingly lower $T$ as $N$ gets larger. For small particle number values $N$, there is a "spike", followed by a rapid decrease. This goes away as $N$ gets closer to the thermodynamic limit.

\subsection{Periodic modulation and static response}

Turning back to the zero-temperature problem, Eq. 1 applies to the general case of a non-interacting gas in an external potential:

$$
\hat{H}=-\frac{\hbar^{2}}{2 m} \sum_{i} \nabla_{i}^{2}+v_{\text {ext }},
$$

where $v_{\mathrm{ext}}=\sum_{i} v\left(\mathbf{r}_{i}\right)$. Although analytical solutions do not necessarily exist, numerical calculations of the energy are easy to implement. We consider the periodic one-body potential $v\left(\mathbf{r}_{i}\right)=2 v_{q} \cos \left(\mathbf{q} \cdot \mathbf{r}_{i}\right)$. This potential is also used when we study NM. For finite $N$ we enforce that a whole number of periods of $v$ span the box length $L$. This is to respect translational invariance. Consequently, calculations of energy at constant density are limited to $N$ that respect this constraint. This can be seen in Fig 4 where the energy per particle at a density of $0.1 \mathrm{fm}^{-3}$ is only plotted at discrete values of $N$. The one-body 


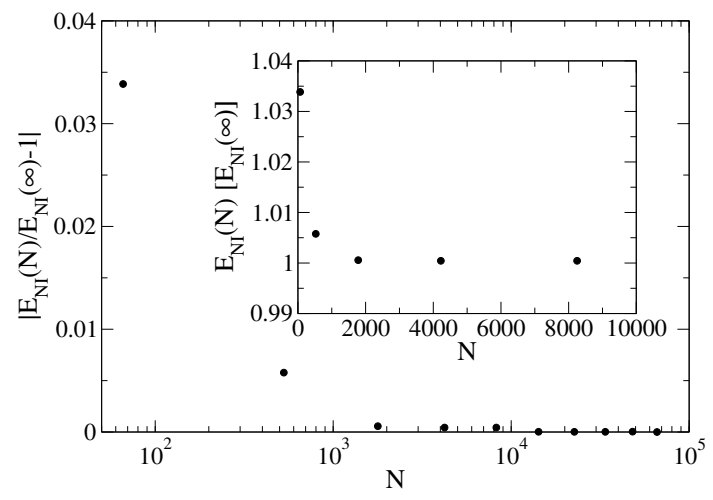

Figure 4. Convergence to the TL for a non-interacting gas in a one-body periodic potential $v\left(\mathbf{r}_{i}\right)=2 v_{q} \cos \left(\mathbf{q} \cdot \mathbf{r}_{i}\right)$. The amplitude is $2 v_{q}=0.5 E_{F}$ and periodicity set so that two periods span the box at the smallest $N$ point. The density is constant at $0.1 \mathrm{fm}^{-3}[12]$.

potential is set to $2 v_{q}=0.5 E_{F}$ and $q=1.4433 \mathrm{fm}^{-3} . E_{N I}(\infty)$ is taken to be approximately $E_{N I}(66000)$ for our calculations. The FS effects are once again seen to die down to 0 as $N$ approaches infinity.

What's nice about the cosine potential is that one can easily extract the linear static response function from energy versus potential strength $v_{q}$. Note that the response function is a property of the unmodulated gas. It quantifies how the system responds to small perturbations. An analytical solution in the TL exists for the non-interacting Fermi gas and is given by the Lindhard function [24] (solid line in Fig. 5). We calculated the response function for 66 and 66000 particles at a density of $0.1 \mathrm{fm}^{-3}$. FS is evident for 66 particles where there is a suppression (squares) compared to the Lindhard function. The 66000 particle response (circles) shows convergence with the TL.

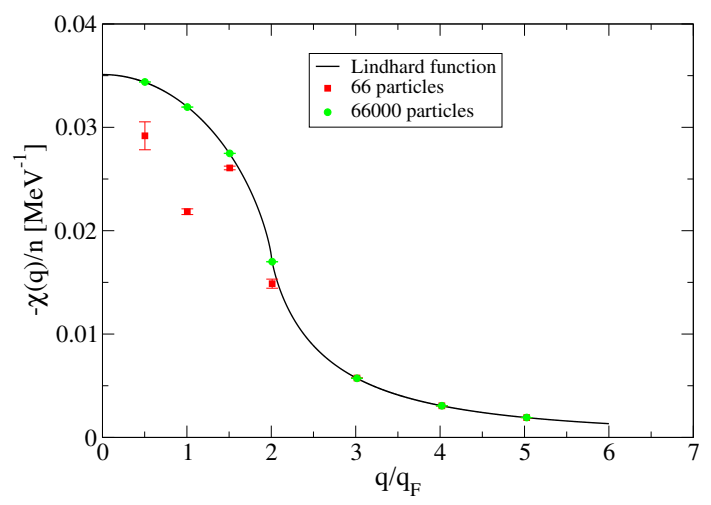

Figure 5. Response functions for the case of the non-interacting Fermi gas (the density is $0.1 \mathrm{fm}^{-3}$ ). Shown are the cases of 66 and 66000 particles, as well as the response in the TL [12]. 


\section{Quantum Monte Carlo}

QMC methods are a stochastic approach to solving the many-body Schrödinger Equation. Most of the algorithms produce exact results for many-body properties. The major exception is for fermions where the fermion-sign problem complicates things significantly. There exist continuous and lattice formulations of the method as well as both zero and finite temperature applications. QMC is very good for studying SC systems up to a decent number of particles. It has been very successful in nuclear physics [25-27]. The number of particles is limited by the computational complexity associated with scaling and is the reason why FS effects are important.

\subsection{Auxiliary Field Diffusion Monte Carlo}

AFDMC is an extension of DMC specialized for handling complicated spinor interactions [28]. The central idea behind the method is to stochastically evaluate the evolution of a trial wave function in imaginary time $\tau:\left|\Psi_{0}\right\rangle=\lim _{\tau \rightarrow \infty} e^{-\widehat{H} \tau}\left|\Psi_{T}\right\rangle$. The excited states decay in this evolution leaving only the ground-state wave function at infinite imaginary time. The ground-state energy is extracted from the statistical data produced in the evolution. Whereas DMC only handles the coordinate degrees of freedom in this fashion, AFDMC treats the spinors stochastically as well.

AFDMC is suitable for the nuclear interaction which contains complicated spin dependence. We use AFDMC to calculate ground-state energies of 66 neutrons. The Hamiltonian contains both twonucleon $(\mathrm{NN})$ and three-nucleon $(\mathrm{NNN})$ interactions:

$$
\hat{H}=-\frac{\hbar^{2}}{2 m} \sum_{i} \nabla_{i}^{2}+\sum_{i<j} v_{i j}+\sum_{i<j<k} v_{i j k}+v_{\text {ext }},
$$

where $v_{\text {ext }}$ is a sum of one-body potentials: $v\left(\mathbf{r}_{i}\right)=2 v_{q} \cos \left(\mathbf{q} \cdot \mathbf{r}_{i}\right)$. The nuclear interaction is strong but also very-short range $(\sim 1 \mathrm{fm})$. This lessens the FS effects in comparison to a longer-ranged interaction. The particles are placed in a box with periodic boundary conditions (just like the noninteracting problem). Furthermore, whenever the evolution produces a coordinate lying outside of the box, the particle reappears at the opposite side of the box. This respects translational invariance and the idea that there are identical boxes filling up all of space. Similarly, when evaluating the interaction part of the Hamiltonian one can consider the interaction between particles in the box and the nearby boxes surrounding it. This helps to mitigate FS effects. Next we look at some results from this method.

\subsection{Equation of State}

We ran simulations for a range of densities and external potential strengths $2 v_{q}$. The Argonne v8' (AV8) and Urbana IX (UIX) potentials were used for the NN and NNN interactions respectively $[27,29]$. This was done for various periodicities of the external potential as well. The energy per particle always falls for increasing potential strength. This makes sense because the particles collect at the troughs of the cosine where the potential is negative. This is visible in Fig. 6 (circles) which depicts AFDMC energies and potential strengths for 66 particles at a density of $0.1 \mathrm{fm}^{-3}$ with $q=4 \pi / L$ (i.e. two periods inside the box). The change in energy with respect to increasing $v_{q}$ is what we refer to as static response. Applying Eq. 1 to these results gives the response for neutron matter. The solid line is energies calculated using SLy4 in the LDA. These results are more closely related to nucleic systems since EDFs are fit to them. The dashed line gives a modified SLy4 where we tuned the isovector term in the EDF to match the energy changes from AFDMC. 


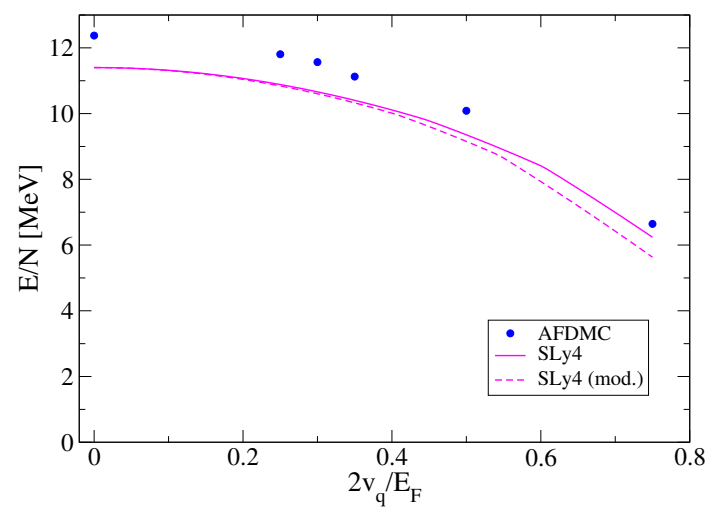

Figure 6. 66 neutron energy per particle versus one-body potential strength with two periods of cosine potential in the box. Circles are AFDMC results with NN+NNN interactions. The solid line depicts SLy4 results from the LDA. The dashed line is SLy4 constrained by AFDMC results. The density is $0.10 \mathrm{fm}^{-3}$ [12].

\section{Conclusion}

We have taken a look at the impact that FS has on quantum mechanical systems. The need to understand these was stressed for SC systems where $N$ is limited by the complexity of the interaction. FS effects in the non-interacting free and periodically modulated gas were investigated, motivating the study of 66 neutrons. It was shown that the much more trivial non-interacting energies provide a FS fix for interacting systems. We then provided an overview of QMC methods which are ideal for studying SC physics. We applied AFDMC to calculations of periodically modulated neutron matter. The results are related to the physics of neutron-star crusts as well as neutron-rich nuclei through the EDF approach.

\section{Acknowledgements}

A. G. would like to thank the Organizers and the Convenors of the Astrophysics, Cosmology and Gravity Session for the invitation and for their efforts on the 6th International Conference on New Frontiers in Physics (ICNFP 2017). This work was supported in part by the Natural Sciences and Engineering Research Council (NSERC) of Canada, the Canada Foundation for Innovation (CFI), and the Early Researcher Award (ERA) program of the Ontario Ministry of Research, Innovation and Science. Computational resources were provided by SHARCNET and NERSC.

\section{References}

[1] J. T. Stewart, J. P. Gaebler, T. E. Drake, and D. S. Jin, Phys. Rev. Lett. 104, 235301 (2010).

[2] O. Juillet, A. Leprévost, J. Bonnard, and R. Frésard, J. Phys. A Math. Theor. 50, 175001 (2017).

[3] S. Gandolfi, A. Gezerlis, and J. Carlson, Ann. Rev. Nucl. Part. Sci. 65, 303 (2015).

[4] K. Hebeler, J. D. Holt, J. Menendez, and A. Schwenk, Ann. Rev. Nucl. Part. Sci. 65, 457 (2015).

[5] B. Friedman and V. R. Pandharipande, Nucl. Phys. A361, 502 (1981).

[6] A. Akmal, V. R. Pandharipande, and D. G. Ravenhall, Phys. Rev. C 58, 1804 (1998).

[7] A. Schwenk and C. J. Pethick, Phys. Rev. Lett. 95, 160401 (2005). 
[8] A. Gezerlis and J. Carlson, Phys. Rev. C 77, 032801(R) (2008).

[9] E. Epelbaum, H. Krebs, D. Lee, and U. -G. Meißner, Eur. Phys. J. A 40, 199 (2009).

[10] N. Kaiser, Eur. Phys. J. A 48, 148 (2012).

[11] M. Buraczynski and A. Gezerlis, Phys. Rev. Lett. 116, 152501 (2016).

[12] M. Buraczynski and A. Gezerlis, Phys. Rev. C 95, 044309 (2017).

[13] M. Bender, P.-H. Heenen, and P.-G. Reinhard, Rev. Mod. Phys. 75, 121 (2003).

[14] E. Chabanat, P. Bonche, P. Haensel, J. Meyer, and R. Schaeffer, Nucl. Phys. A 635, 231 (1998).

[15] R. Sellahewa and A. Rios, Phys. Rev. C 90, 054327 (2014).

[16] C.J. Yang, M. Grasso, D. Lacroix, Phys. Rev. C 94, 031301(R) (2016).

[17] S. A. Fayans, JETP Lett. 68, 169 (1998).

[18] B. A. Brown, Phys. Rev. Lett. 855296 (2000).

[19] F. Chappert, M. Girod, and S. Hilaire, Phys. Lett. B 668, 420 (2008).

[20] F. J. Fattoyev, C. J. Horowitz, J. Piekarewicz, and G. Shen, Phys. Rev. C 82, 055803 (2010); F. J. Fattoyev, W. G. Newton, J. Xu, B.-A. Li, Phys. Rev. C 86, 025804 (2012).

[21] B. A. Brown and A. Schwenk, Phys. Rev. C 89 011307(R) (2014).

[22] E. Rrapaj, A. Roggero, J. W. Holt, Phys. Rev. C 93, 065801 (2016).

[23] W. G. Dawkins and A. Gezerlis, Phys. Rev. A 96, 043619 (2017).

[24] C. Kittel, Solid State Physics, edited by F. Seitz, D. Turnball, H. Ehrenreich 22, 1, (Academic Press: New York, 1968).

[25] S. Gandolfi, J. Carlson, and S. Reddy, Phys. Rev. C 85, 032801 (2012).

[26] A. Gezerlis, I. Tews, E. Epelbaum, S. Gandolfi, K. Hebeler, A. Nogga, and A. Schwenk, Phys. Rev. Lett. 111, 032501 (2013).

[27] B. S. Pudliner, V. R. Pandharipande, J. Carlson, S. C. Pieper, and R. B. Wiringa, Phys. Rev. C 56, 1720 (1997).

[28] K. E. Schmidt and S. Fantoni, Phys. Lett. B 446, 99 (1999).

[29] R. B. Wiringa and S. C. Pieper, Phys. Rev. Lett. 89, 182501 (2002). 